\title{
Luciano Rocchi
}

\section{Il glossario italo-turco contenuto in un codice fiorentino del XVI secolo}

DOI 10.1515/zrp-2016-0120

Abstract: The codex Ashb. 1547 of the Biblioteca Mediceo-Laurenziana of Florence contains a substantial lexical and phraseological Italian-Arabic glossary, with two additional short chapters regarding Greek and Turkish. The manuscript, unpublished so far, probably dates from the 16th century and is anonymous; from the internal data, we can infer that its author was a merchant of Venetian origin, who lived in Egypt for a while. It has to be pointed out that the texts in the various languages are all written only in the Latin script. This paper deals with the Italian-Turkish part of the codex, which comprises words and phrases concerning greetings and buying and selling, as well as a series of numerals. The Turkish lexical corpus is first presented in alphabetical order, if necessary comparing single entries to those of other Transkriptionstexte or of literary Ottoman; in a second step its graphematic, phonetic and morphological features are examined.

Keywords: historical lexicography, Ottoman-Turkish, Italian

Parole chiave: lessicografia storica, turco-ottomano, italiano

\section{Il codice laurenziano Ashb. 1547}

1.0 Nella Biblioteca Mediceo-Laurenziana di Firenze è conservato (coll. Ashb. 1547) un codice manoscritto cartaceo, in $12^{\circ}$, che nell'Index Codicum Manoscriptorum Orientalium della stessa biblioteca è catalogato col titolo Vocabolario italiano e arabesco, con alcuni Dialoghi in turchesco e in greco moderno e datato al XVI secolo. Essendo entrati recentemente in possesso della riproduzione fotografica dell'intero manoscritto, possiamo affermare che in realtà esso non reca alcuna titolatura generale al suo inizio (del resto era palese che la dicitura che si legge nel catalogo non poteva essere tratta dal codice, perché è inimmaginabile che nel '500 si usasse l'espressione greco moderno). Il manoscritto, composto di 165 fogli,

Indirizzo di corrispondenza: Prof. Dr. Luciano Rocchi, Università degli Studi di Trieste, Dipartimento di Scienze Giuridiche, del Linguaggio, dell'Interpretazione e della Traduzione, via Filzi 14, I-34132 Trieste, E-Mail: Irocchi@units.it 
comprende un sommario del contenuto (Tavola de gli Capitoli, ff. 2b-3b), un repertorio lessicale e fraseologico italiano-arabo (Vocaboli italiani et arabeschi, si legge nell'intestazione del primo capitolo) suddiviso in ventinove capitoli ciascuno dei quali si occupa di un determinato campo semantico (ff. 5a-111a; i ff. 111b$113 \mathrm{~b}$ sono vuoti), una lunga lista di forme verbali italiane e arabe (Significato d'molti verbi, ff. 114a-139b), una lista di parole arabe omofone o quasi-omofone coi loro rispettivi significati italiani (Capitolo trentesimo nel qual tratta di uarietà o significato diuerso de uocaboli simili o quasi simili, ff. 140a-149b), una breve parte dedicata al greco (Capitolo trentesimo primo tratta di salutar' cõprar' e' uẽder' et numerare ĩ lingua italiana e greca, ff. 150a-153b), una dedicata al turco (su cui vedi §2), una specie di colofone di cui parleremo al \$1.1 (ff. 157b-159a), e infine una lista dei verbi italiani presenti nella parte italiano-araba (Tavola d'gli Verbi, ff. 160a-164a) con rinvio al numero di pagina, in quanto l'autore ha numerato (da $1 \mathrm{a} 310$ ) le pagine che vanno da f. $5 \mathrm{a}$ a f. 159b. Va ancora detto che parecchie volte mancano i corrispondenti arabi (e anche qualcuno greco) delle parole e frasi italiane e addirittura il capitolo dodicesimo, che avrebbe dovuto illustrare la terminologia nautica (tratta de nauigli, naue, galere, et altre cose, ff. 52a-53b), è lasciato interamente in bianco, nei riguardi sia del lemmario italiano sia di quello arabo. Tutti i testi nelle varie lingue (arabo, greco, turco) sono redatti nel solo alfabeto latino.

1.1 Al termine dei trentadue capitoli, prima della conclusiva Tavola dei verbi italiani, vi sono due carte senza intestazione e non elencate nell'indice iniziale, che sembrano contenere un colofone bilingue, caratterizzato però da alcune peculiarità. Il testo arabo ha una traduzione italiana interlineare, in modo che ogni vocabolo o sintagma arabo rechi sotto di sé l'equivalente italiano. Qui di seguito riportiamo tale traduzione, che, per i motivi suddetti, ricalca l'arabo, lessicalmente e sintatticamente, commentandone di volta in volta i singoli passi: $^{1}$

(157b) io nõ so parlar arabo, io ne sapeua un poco mi sõ smẽ(tica)to ogni cosa, adesso nõ so mãco intẽdo, e se ho fatto il libro bono, ho auuto mestro bono arabo; io ui dico che non intendo il nostro autore parla evidentemente di sé e della sua opera, anche se è sorprendente, rispetto ai colofoni usuali, che taccia il suo nome, forse per professione di modestia, vista la sua ostentata dichiarazione di ignoranza dell'arabo;

1 È mantenuta la grafia originale, con la sola aggiunta di qualche accento e apostrofo. Le integrazioni apportate a forme abbreviate o errate sono poste tra parentesi. Rendiamo con $\sim$ il simbolo di separazione usato dall'autore. 
se io nõ faccio dispiacere, sto gu(a)rdãdo o io guardo, se uedo cosa che mi piacia per acomprare, e se nõ o e se (158a) nõ uedo niente me ne $u($ ad)o a mia strata $\sim$ è probabilmente un riferimento alla sua attività di mercante; che tale fosse il mestiere del Nostro risulta in modo indubitabile dalla straordinaria ricchezza terminologica dei capitoli riguardanti i beni di cui si faceva commercio: spezie e droghe (circa 130 lessemi, tra cui cinque specie di zenzero e altrettante di pepe), pietre preziose (una quarantina), metalli (una cinquantina, tra cui, a seconda dei vari tipi di lavorazione, otto concernenti l'oro, nove l'argento, dieci il rame), tessuti (una cinquantina). Del resto è assai indicativo che i due capitoletti scelti per il greco e il turco siano relativi soprattutto al «comprare e vendere»;

io sto nela cõtrada di beĩ surein presso la uolta nel loco che coceno dẽtro il zucaro a tua mano drita la casa noua biãca giusto giusto ĩ mezo tra la beueratora e la uolta dou'è la casa tua doue stai viene indicata l'ubicazione della casa dove presumibilmente risiedeva, sita in Beyn es-Sureyn ('Tra le Mura'), una nota strada del Cairo. Un'ulteriore conferma del luogo del suo soggiorno in Oriente viene data dall'intestazione del ventiduesimo capitolo: tratta de pesi e misure di Egitto. Naturalmente non si può escludere che abbia viaggiato anche altrove nell'area del Mediterraneo orientale;

(158b) questa nõ è la strata del signore in la strada del signore uoi mi forziarmi noi stiamo nel deserto ricordati ch' siamo in cairo nel loco di iusticia il signor mi a sigurato nel suo paese e tu tieni core di spoiarmi e batermi e amazarmi o signori siati testimoni come questo mi uole forcia(r)mi e leuarmi (159a) sig(n)or mai ti ò cognosiuto et io cõ bon amor t’ò datto [sic] la mia roba a (c)redẽza mi doueresti render gratia $\tilde{\imath}$ pagar il debito ch' mi doueti or'mai seria tẽpo cõ la cortesia o cõ la iusticia già aueti saputo come ho perso mia roba in mare per fortuna soto la forteza di alessãdria adesso mi gu(a)dagno il pane alla giornata cõ trauaglio, ricordatiue la morte $\sim$ non sappiamo se le vicissitudini qui narrate in forma allocutiva riflettano tristi esperienze personali o se si tratti di una semplice esercitazione letteraria.

1.2 Circa la sua provenienza, il nostro mercante era quasi certamente di origine veneziana o veneta. Infatti, anche se la lingua che usa si rifà nel suo complesso al modello toscano, ogni tanto ne emergono pretti dialettalismi veneziani (cercio 'cerchio (della botte)', ciodo 'chiodo', fregole di pane 'briciole', intaiador 'intagliatore', madona 'suocera', nonanta 'novanta', pesse 'pesce', pulisi 'pulci', ecc.; si noti anche la forma spoiarmi in uno dei passi succitati). Significativa è inoltre la costante incertezza grafica tra geminazione e scempiamento delle consonanti, e ci pare notevole un bel caso di ipercorrezione toscaneggiante dell'approssimante [j] (il cane abaglia). 
1.3 La datazione del codice al XVI sec. ${ }^{2}$ assegnatagli dal Catalogo appare senza dubbio verosimile. Come s'è già sopra accennato, la lingua impiegata dall'autore appartiene alla koinè di tipo toscano o toscaneggiante che in epoca cinquecentesca si era ormai affermata: "Quella che nei secoli precedenti era un'attività individualmente sviluppata dai singoli su sostrati regionali diversi, diventa nel Cinquecento un'attività dominata da correnti di gusto collettivo [...] e da norme grammaticali che ottengono larghi consensi. [...] Una notevole uniformità nella lingua scritta si sta attuando tra le persone colte» (Migliorini 1978, 332). Altro dato importante è che venga usato, al posto di volgare o toscano, il termine italiano: è solo nel Cinquecento che «questa espressione guadagna terreno» (id., 360 n. 1). Dal punto di vista grafico, l'autore non si serve del consueto corsivo manuale, bensì imita la scrittura dei testi a stampa, il che, se da un lato facilita la lettura - ma $\langle\mathrm{e}\rangle \mathrm{e}<\mathrm{c}\rangle$ si confondono spesso, e talora $\langle\mathrm{b}\rangle$ e $\langle\mathrm{h}\rangle-$, dall'altro non aiuta certo una datazione del manoscritto basata su criteri paleografici.

1.4 In definitiva, siamo dell'opinione che questo codice laurenziano rappresenti una testimonianza di eccezionale valore per la lessicografia e dialettologia storica araba, in virtù sia della sua antichità sia della resa in alfabeto latino sia del copioso materiale fornito.

\section{Il capitolo «in lingua italiana e turchesca»}

Il trentaduesimo capitolo del manoscritto reca l'intestazione Capitolo trentesimo secõdo tratta di salutar cõprar' e' uẽdere et numerare ĩ lingua italiana e turchesca (ff. 154a-157a; pp. 299-305 nella numerazione dell'autore) ed è formato da 74 frasi e 53 numerali in redazione bilingue italo-turca. A quanto ci risulta, esso è ancora inedito, eccezion fatta per alcune frasi ivi contenute che Manlio Cortelazzo ha citato (senza commento linguistico) in un suo lavoro dedicato alla conoscenza del turco nell'Italia del sedicesimo secolo $(1979,135)$. Rispetto agli altri specimina di lingua turca che si riscontrano negli opuscoli e manuali poliglotti che circolavano allora in Italia in discreto numero (Cortelazzo 1979; Rossebastiano 2000, 689s.) e che presentano in genere una veste grafica molto approssimativa, il nostro glossario fa decisamente migliore figura: non appare certo ricopiato da qualche fonte precedente e l'autore l'ha verosimilmente compilato sulla base di informazioni dirette ricevute da parlanti turchi. È quindi un tassello di valore non marginale che va ad aggiungersi alla serie di quei testi in trascrizione la cui importanza per lo studio del turco-ottomano risulta sempre più evidente.

2 In Rossebastiano $(2000,690)$ si legge «XVe siècle», ma si tratta prob. di un errore di stampa. 


\section{Corpus lessicale turco}

Il testo turco viene riportato sia in grafia normalizzata, basata sull'alfabeto moderno, con adeguamenti formali più o meno sensibili al turco-ottomano letterario, sia nella grafia originale, tra parentesi tonde. Il testo redatto in italiano è mantenuto inalterato nella sua veste grafica, tranne che per l'inserimento di accenti e apostrofi secondo l'uso moderno (si noti come l'autore tralasci il punto interrogativo nelle domande); le abbreviazioni sono sciolte tra parentesi. Se ci è parso il caso, abbiamo aggiunto a certi lemmi brevi commenti illustrativi, introdotti dal simbolo •. Per le osservazioni linguistiche di carattere generale cf. $§ 4 .{ }^{3}$

acca/açça çıkar acca (cicar aggia) ‘cacciati moneta' (155b), say aççayı (sai aciai) 'cõta dinari' (155b) • Cf. ant. osm. ağca 'akçe, Osmanlı küçük sikkesi ve para birimi’ (Ferec [1451]: TETTL 1, 115), açça (< akça) (accia) ‘aspro’ (Argenti: Rocchi 2007, 26, con ulteriori attestazioni della forma assimilata). Per la discussione etimologica sulla parola cf. Rocchi 2011, 53s.

aç- $\rightarrow$ kese.

açça $\rightarrow$ acca.

al- al (al) 'piglia'(155b); alırum (alerun) 'uoglio cõprare' (154b); alır misin (alermisen) 'uoi comprare' (154b); $\rightarrow$ satm al-.

alek, aleykum $\rightarrow$ sälam.

Allah $\rightarrow$ ısmarla-.

altı (alti) 'sei' (156a); $\rightarrow$ igrimi, on .

altmış (altmis) 'sesanta' (157a).

altun bir altun (bir altun) 'uno ducato' (154b) • Osm. altun 'aureus, ducatus' (Men. 381).

ben $\rightarrow$ satin al-.

bereket bereket versin (berichet uersin) 'grã mercè' (155a) • Tml. bereket versin 'Dio vi conceda l'abbondanza! f[o]rm[ula] di ringraziamento nel ricevere il denaro dopo un contratto, una vendita, ecc.' (AngSm. 97), osm. lett. berekât virsün 'Deo gratias. Forma gratias agendi post prandium, aut donum acceptum' (Men. 780).

beş (bes) 'cinque' (156a); $\rightarrow$ igrimi, on .

bin (bin) 'mile o mila', iki bin (ichi bin) ‘dui mila', üç bin (ug bin) 'tre mila', yüz bin (ius bin) 'cento mila', iki yüz bin (ichi ius bin) 'ducẽto mila’, üç yüz bin (ug ius bin) 'trecento mila', bin keret bin (bin chieret bin) 'vno millione' [propr.

3 Le abbreviazioni concernenti le lingue sono le seguenti: ant. = antico, lett. = letterario, osm. = osmanl, $\mathrm{t}$. turco, tml. = turco moderno letterario. 
'mille volte mille'] (157a) • Cf. la resa meninskiana di 'millione': on kerre yüz bin propr. 'dieci volte centomila' (Men. On. 1049).

bir (bir) 'vno' (156a); $\rightarrow$ altun, daha, igrimi, on, otuz.

bu bu eyi (boei) ‘questo è bono', 'questa è bona', bunlar eyi (bonarei) 'questi sono boni', 'queste sõ bone' (155a); $\rightarrow$ iste- • Per il vocalismo /o/ cf.<boni $>=$ bunu in Lupis (4) = Adamović (1975, 237), <bohalton> = bu altun 'questo scudo' nel Vocabulario Nuovo (Adamović 1976, 62). Per l'assimilazione della laterale nella forma di plurale cf. t. dial. anatolico bunnar 'bunlar' (TTAS).

bul- $\rightarrow$ safa.

bunda işte bunda (stebõda) 'ecola qua' [risposta a 'dà qua la roba' $\rightarrow$ geter-] (155b), bundayım (bondain ${ }^{4}$ ) 'ecome qua' (154b) • Cf. bundayum (bundaium) 'eccomi' (Carradori: Rocchi 2011, 90). Per il vocalismo /o/ la forma bonda è attestata in B. Georgievits (Heffening 1942, 26) e nel Vocabulario Nuovo (Adamović 1976, 56).

çek- çekme (giechme) 'non stirare' (155b).

çelebi ya çelebi (ia celebi) ‘o gentil homo’ (155b).

çıkar- $\rightarrow$ acca/açça.

çok $\rightarrow$ pahalı • Cf. ant. osm. çoh 'çok' (TS 933s.).

daha $\rightarrow$ keret, say-.

de- dedüm (dedun) 'ho detto' (155a).

doksän (dochsen) 'nonãta' (157a).

dokuz (docus) 'noue' (156a); $\rightarrow$ igrimi, on .

dört (dort) ‘quattro' (156a); $\rightarrow$ igrimi, on, yüz.

elli (eli) ‘cinquanta' (156b).

ender- enderme tereziyi (ender me terezii) 'nõ appogiar la bilãza' (155b) • Cf. ender- (osm. lett. endür-/endir-, tml. indir-) 'discarico, metto abbasso, fo scendere, scarico’ (Argenti: Rocchi 2007, 94).

espap $\rightarrow$ geter- • Cf. espap (osm. lett. esvab, tml. esvap) 'roba' (Argenti: Rocchi 2007, 96).

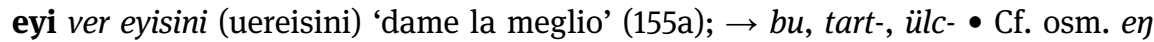
eyüsi 'il migliore' (Men. 618), tml. iyisi 'il buono, il migliore, il meglio' (AngSm. 397).

gel- gele gele (giele giele) 'uieni uieni’ (155b); $\rightarrow$ safa • Il suff. -a/-e «eskiden, pekitmek için emir kipinin tekili sonuna eklenirdi» (TS 7, 3).

gerek vermem gerek ( ${ }^{\star}$ uerme gierech) 'la *uoglio dare' (155b) • La frase turca esprime ovviamente la necessità: 'devo dare'. Abbiamo rettificato il verme del codice in base alla forma di prima persona della versione italiana.

4 Cortelazzo $(1979,135)$ legge erroneamente hondain. 
geter- geter espapı (gieteres papi) 'dà qua la roba' (155b) • La forma geter- (osm. lett. getür-, tml. getir-) è attestata sia anticamente (Argenti, Ferraguto, Carradori) sia nel t. dial. odierno (Rocchi 2012, 87).

git- gidelüm (o forse gidelım) (chidelũ) 'andiamo' (155b) • Cf. t. dial. kitmek 'gitmek' (TTAS).

hal nedir (-dür) halın (nedr alen) 'come stai', nedır (-dür) halınuz (nedr alenus)

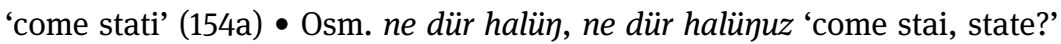
(Men. 1712s.). Il grafema meninskiano <ü> può rappresentare anche il suono [u] nelle parole a vocalismo velare (Stachowski 2012).

hani $\rightarrow$ usta • Osm. kani, vul. hani 'ubi, ubi tandem' (Men. 3773), tml. hani 'e dove? dove mai?' (AngSm. 316).

hizmet senin hizmetine (senen chesmetene) 'al u(ost)ro comãdo' (154a) • Propr. 'al tuo servizio'. È incerto se il nostro dato sia da leggere hızmet o kızmet (cf. kizmet 'service' in Preindl 1790, 533).

1smarla- yahu Allaha ısmarladık (iau alla casmar ladic) 'me recomãdo' [formula di commiato] (154b).

igrimi (icrimi) 'vinti’, igrimi bir (icrimi bir) ‘uinti uno', igrimi iki (icrimi ichi) 'uinti dua', igrimi üç (icrimi ug) 'uintitre', igrimi dört (icrimi dort) 'uinti quattro', igrimi beş (icrimi bes) 'uinti cinque', igrimi altı (icrimi alti) 'uinti sei' igrimi yedi (icrimi iedi) 'uinti sette', igrimi sekiz (icrimi sechis) 'uinti otto', igrimi dokuz (icrimi docus) 'unti noue' (156b) • La forma igrimi è attestata in Lupis (6) = Adamović (1975, 240) e in Argenti (Rocchi 2007, 127).

iki (ichi) 'dui' (156a); $\rightarrow$ bin, igrimi, on, otuz, yüz.

iste- bu kadar isterüm (bucadar isterũ) 'tanto ne uoglio' (155a), ne istersin (neisterssin [nestersin (155b)]) 'che uolete' (154a), 'che uoi' (155b), ne kadar istersin buna (necadar istersĩ bonà) ‘quãto uoi di questo’ (154a).

işte $\rightarrow$ bunda.

kadar $\rightarrow$ iste -

kavl et- kavl edelım (caul edelem) 'acordiamosi', 'famo patto', kavl ettık (`caulet etech) 'ho fatto patto' (155a) • La traduzione italiana alla prima persona singolare nell'ultimo esempio si spiega supponendo l'ellissi di una forma comitativa: (seninle/onunla) kavl ettik 'ho fatto patto (con te/lui)'.

kayl kaylım (cailem) ‘sono cõtento', kayl misın (cail misin) ‘sei cõtento’ (155b).

keremet keremet eyle (cromet eile) 'fami piacere' (154b); keremet ettüm (cromet etun) 'ui facio apiacere' (155a) • Cf. osm. kerem eyle 'di grazia, vi prego, fate grazia' (Men. 3924), kerem et- 'to have the kindness to (so and so)' (Redhouse 1890, 1540). Il dato del nostro autore denota contaminazione con l'altro arabismo, corradicale e parzialmente sinonimo, keramet 'generosità, munificenza, dignità' (Men. 3901). Per l'aspetto fonetico §§4.2.0.1, 4.2.0.2. 
keret satın al bir keret daha (satin albir cheret daa) 'cõpra un'altra uolta’ (154b); $\rightarrow$ bin.

kese $a c ̧$ keseyi (atcs ${ }^{5}$ chesei) 'apri la borsa' (155b).

kurk (crich) 'quaranta' (156b) • Per la forma metatetica cf. le grafie $<$ chrich $>$ (Lupis 7) $=<$ cric $>$ (Adamović 1975, 221), <chruch $>$ (Vocabulario Nuovo: Adamović 1976, 51).

mazur mazur tutun (maazur tuntun) 'perdonatime' (154a) • Cf. mäzur tut 'compatitemi, riceuete con buon'animo, gradite' (Men. 4764). La grafia <aa> riflette senza dubbio, come indicano pure altri testi in trascrizione, la presenza dell' 'ayn nella parola araba fonte del prestito ( $m a$ 'dūr).

ne $\rightarrow$ hal, iste-, verecek ${ }^{\star}$.

nola (nola) 'volontera' (154b) • Osm. nola 'fia, sì bene, volontieri' (Men. 5279). \$4.3.1.0.d.

on (on) 'diece', on bir (on bir) 'undeci', on iki (on ichi) 'dodeci', on üç (on ug) 'tredeci', on dört (on dort) 'quatordeci', on beş (on bes) 'quindeci', on altı (on alti) 'sedeci' (156a), on yedi (on iedi) 'diecesette', on sekiz (on sechis) 'dieceotto', on dokuz (on docus) 'diecenoue' (156b).

otuz (otus) 'trenta', otuz bir (otus bir) 'trẽta uno', otuz iki (otus ichi) 'trenta dua', otuz üç (otus ug) 'trenta tre' (156b).

pahalı çok pahalı (gioh paali/paale) 'è troppo cara', 'son troppo care' (155a).

pek sen yauz peksin (sẽ iaus pecsin) 'tu sei molto stretto' [= avaro] (155a) • Osm. pek 'parco, scarso', eli pek 'duro, scarso di mano' (Men. 859), tml. pek tutmak 'essere spilorcio, taccagno' (AngSm. 663).

saät saät salamet (saet salamet) 'alla bonora' (154b) • Cf. la grafia meninskiana <sā-æt>. Va detto che l'aspetto morfologico del sintagma è poco chiaro; probabilmente occorre emendarlo con l'aggiunta del comitativo (i)le: cf. selametle/selamet ile 'cum salute \& incolumitate, incolumiter' (Men. 2651).

safa safa geldin (safa ieldin) 'il ben uenuto', safa geldinüz (safa ieldinus) 'siati li ben uenuti', safa *buldun (safa boldun) 'il ben trouato', safa *buldunuz (safa boldunus) 'siati li bẽ trouati' (154a) • La risposta turca usuale a safa geldin(iz) è safa bulduk. È palese che nel dato del codice laurenziano c'è stata contaminazione con le forme del verbo gel-. Un processo analogo si riscontra in Argenti, dove le due espressioni formulari suonano safa guldun e safa buldun (Rocchi 2007, 205).

sağlık var sağlığla (uar saghligla/saghlighla) 'ua alla bon'ora', 'andate cõ Dio' (155b) • Osm. var sağliğle 'và sano, addio, và con Dio’ (Men. 2920).

5 In teoria si potrebbe leggere anche <ates>, il che ovviamente è da escludere. In ogni caso la grafia $<\mathrm{tcs}>$ per rendere l'affricata postalveolare sembra un unicum nel manoscritto. 
sälam salamalek (salamalech) 'salute sopra di te', 'bondì o bona notte', as-salamu aleykum (asalamu aleicum) 'salute sopra di uoi', alek selam (alechselam) 'sopra di te la salute', aleykum as-salamu (aleicum asalamu) 'sopra di uoi la salute' (154a) • Si noti la forma prettamente araba as-salamu mantenuta dall'autore.

salamet $\rightarrow$ saät.

sat- satarmm (satáran) 'uoglio uender', satar misın (satar misin) 'uoi uender' (154b).

satın al- satın al (satinal) 'acompra', ben satın alırum (ben satin alrun) 'io acompro', ben satın aldum (ben satin aldun) 'ho comprato', satın alayım (satin alain) 'acomprarò' (154b); $\rightarrow$ keret.

say- sayırum (sairun) 'conto' (155b), 'contarò' (156a), saydum (saidun) 'ho contato’, say bir daha (sai bir daa) 'cõta ũ altra uolta' (156a); $\rightarrow$ acca/açça.

sekiz (sechis) ‘otto' (156a); $\rightarrow$ igrimi, on .

seksen (sechsen) 'ottanta' (157a).

sen $\rightarrow$ pek.

senin $\rightarrow$ hizmet.

tamam $\rightarrow$ ülc-.

tart- eyi tart (eitart) ‘fa bon peso' (155b).

terezi $\rightarrow$ ender- $\bullet$ La var. terezi (osm. lett. terazu, tml. terazi) è attestata anche in Argenti (Rocchi 2007, 241, con ulteriore documentazione).

tut- $\rightarrow$ mazur .

ucuz (ugius) ‘è bõ mercato’ (155a).

usta hani usta (ani usta) ‘dou'è il mestro' (154b).

üç (ug) 'tre' (156a); $\rightarrow$ bin, igrimi, on, otuz, yüz.

ülç- eyi ülç (eiulg) 'fa bona misura', tamam ülç (tamen ulg), ülç tamam (ulg tamam) 'misura giusto' (155b) • La var. ülç- in luogo del letterario ölç- è ampiamente attestata nei Transkriptionstexte (Argenti, Vocabulario Nuovo, Megiser, Ferraguto, Carradori). La forma <tamen> è molto sospetta e va forse emendata in <teman> (attestato in J. Nagy de Harsány: Hazai 1973, 261).

var- $\rightarrow$ sag $l l k^{*}$.

ver- verir misin (uerir misin) 'uoila dare', vermezüm (uarmezũ), veremem (ueremẽ) 'nõ la uoglio dare' (155a); $\rightarrow$ bereket, eyi, gerek.

verecek` vereceğin nedır (-dür) (ueregie inedr) ‘quãto l'ultimo’ (155a) • Tml. verecek 'debito, dare' (AngSm. 872). La frase italiana dovrebbe intendersi 'quant'è il tuo ultimo debito?, quanto ti resta da pagare?'. ya $\rightarrow$ çelebi.

yahu (iau) 'adio' (154a); $\rightarrow$ ssmarla- • Osm. yahu 'vale, adio' (Men. 5556).

yauz $\rightarrow$ pek • Osm. yauz 'cattivo, sdegnato, fiero, furioso, crudo, brusco' (Men. 5555), tml. yavuz 'duro, severo; audace, bravo; buono, eccellente' 
(AngSm. 893). L'uso del vocabolo come semplice rafforzativo ('molto, assai') da parte del nostro autore trova buone corrispondenze in F. Argenti, cf. yauz incinür ki bizüm hizmetkârlar öyle ettiler (iaus inginur chi bisum chesmetchiarlar oile ettiler) 'molto ha per male che i nostri seruitori così habbino facto' (Bombaci 1938, 21), yauz ev- (iaus euerum) 'ho fretta' [propr. 'ho molta fretta'] (Rocchi 2007, 264).

yedi (iedi) 'sette' (156a); $\rightarrow$ igrimi, on .

yetmiş (ietmis) 'settanta' (156a).

yüz (ius) 'cento', yüz bir (ius bir) 'cento uno', yüz iki (ius ichi) 'cento dui', yüz üç (ius ug) 'cento tre', iki yüz (ichi ius) 'ducẽto', üç yüz (ug ius) 'trecẽto', dört yüz (dort ius) ‘quattro cẽto' (157a); $\rightarrow$ bin.

\section{Osservazioni linguistiche}

I dati del glossario sono citati nella grafia originale. Il simbolo $\rightarrow$ rinvia ai lemmi in grafia normalizzata del corpus.

\subsection{Grafematica}

Le parole turche vengono trascritte dall'autore in alfabeto latino secondo le consuetudini ortografiche italiane, come del resto era logico aspettarsi. Diamo qualche cenno sulla resa dei suoni estranei al sistema fonologico italiano:

$l^{6}$ per lo più <i $>$ : alti, altmis, crich ( $\rightarrow$ krk), saghlig(h)la, satinal, ecc.; talvolta anche $\langle\mathrm{e}\rangle$ : alen $(\rightarrow$ hal), aler misen, cailem, chesmetene, edelem, senen; raramente $\langle\emptyset\rangle$ : alrun. In un paio di casi una stessa parola ha grafie diverse relativamente a questa vocale: alerun alrun, paali paale. Per satáran, nedr cf. §4.2.0.1.

$\ddot{o}<0>$ : dort, cromet $(\rightarrow$ keremet; \$4.2.0.1).

$\ddot{u}<\mathrm{u}>$ : dedun, ug, ulg.

$h[\mathrm{x}]<\mathrm{h}>$ : gioh $(\rightarrow$ çok); possibilmente $<\mathrm{ch}>$ in chesmetene, ma il digrafo potrebbe anche indicare una occlusiva (cf. il commento a $\rightarrow$ hizmet), come è la norma

6 Il grafema $\imath$ denota la vocale alta posteriore non arrotondata [u] (Stachowski 2012, 189), ma secondo diversi lavori scientifici, anche di buon livello, essa sarebbe invece articolata più centralmente e viene quindi foneticamente trascritta [i] (p. es. Wendt 1987, 317; Csató/Johanson 1998, 207). Anche il Duden $(1974,115)$ nota la vocale turca come [i] , aggiungendo però «ist weiter hinten, gegen $[\mathrm{wu}] »$. Secondo Ergenç $(2002,57)$ «the /1/ vowel that is listed as a back vowel is also viewed as medium vowel (= ortadil ünlüsü) because of its location of origin». 
nelle altre parole. Come si sa, questo suono è scomparso dal t. moderno standard e sopravvive solo a livello dialettale.

La fricativa sonora [z] è resa $<\mathrm{z}>$ in posizione intervocalica: maazur, terezii, uarmezũ ( $\rightarrow$ ver-), ma sempre $<\mathrm{s}>$ a fine di parola: alenus, docus, iaus, ieldinus, sechis, ecc. Può anche darsi che ciò indichi un assordimento della consonante in posizione finale.

Per il singolare trigrafo $<\mathrm{tcs}>\mathrm{cf}$. n. 5 .

\subsection{Fonetica/fonologia}

\subsubsection{Vocalismo}

\subsubsection{Modificazioni del timbro}

(e $>$ i) berichet.

$(1>$ a) satáran. Sembra un'assimilazione vocalica a distanza.

(e > ö) cromet. La labializzazione della vocale è senza dubbio dovuta alla vicinanza di $[\mathrm{m}]$.

$(\mathrm{u}>\mathrm{o})$ bo, bonda.

Due casi si presentano problematici:

1) La forma nedr, ossia il pronome ne + la copula $d I r / d U r$, corrisponde al t. comune nedir (osm. anche nedür). L'assenza di un grafema vocalico fra $<\mathrm{d}>\mathrm{e}$ $<\mathrm{r}>$ parrebbe indicare però che il dato del codice vada letto [nedur] (cf. alrun). Si possono prospettare due ipotesi: a) si tratta effettivamente di nedir, con netta difformità dalle regole dell'armonia vocalica, cf. edelem (= edelım), senen (= senin); b) oppure di un nedir/nedür con vocale velarizzata, o perlomeno percepita come tale dal nostro autore in base alla pronuncia del suo informatore.

2) La <a $>$ di uarmezũ ( $\rightarrow$ ver-) è molto strana, non solo perché la $e$ di ver- è di norma chiusa, ma anche perché altrove si trova scritto sempre <uer->. Forse mero errore grafico.

\subsubsection{Sincope}

Un solo esempio, nella prima sillaba: cromet. 


\subsubsection{Aferesi}

I casi di caduta dell'iniziale vocalica si verificano davanti al nesso $s+$ consonante: smarladic, ste $(\rightarrow$ işte), ne stersin (accanto al regolare ne istersin).

\subsubsection{Consonantismo}

\subsubsection{Sonorizzazione/desonorizzazione}

$(\mathrm{g}>\mathrm{k})$ icrimi, chidelũ $(\rightarrow$ git-).

(ç > c) Attestato sia all'inizio di parola: giechme ( $\rightarrow$ çek), gioh $(\rightarrow$ çok), sia alla

fine: üc, ülc. La doppia forma $\rightarrow$ acca $\sim$ açça potrebbe non riguardare questo fenomeno, ma risultare da basi ottomane diverse (cf. il commento al lemma).

Per la possibile perdita di sonorità di [z] in posizione finale cf. supra 4.1.

\subsubsection{Palatalizzazione}

( $\mathrm{g}>\mathrm{c}, \mathrm{y}$ ) Regolarmente davanti a e: giele, gierech, gieter, ieldin(us).

\subsubsection{Altre modificazioni consonantiche}

$(\mathrm{n}>\mathrm{n})$ La nasale velare dell'osmanlı letterario appare sempre come una nasale alveolare, almeno a livello grafico: alen(us), bin, bona, ieldin(us), senen, tuntun. $(\mathrm{m}>\mathrm{n})$ Molto spesso le forme verbali di prima persona presentano una nasale finale alveolare al posto della bilabiale: aldun, al(e)run, bondain, dedun, etun, saidun, sairun, satáran. È un fenomeno ben documentato in antico e medio osmanlı (TS 7, 128-134); per la forma di ottativo alain cf. però §4.3.0.1.d. Un altro esempio di questo passaggio lo si trova in tamen (forma dubbia, cf. il commento a $\rightarrow$ ülç-), ma qui entra prob. in gioco un processo dissimilatorio. (ş > s) Regolarmente in tutti gli esempi: altmis, bes, ietmis, ste (= işte).

$(\mathrm{h}>\mathrm{k})$ Un solo caso in allaca $(\rightarrow$ ssmarla-). Di norma questo suono si dilegua, §4.2.1.4.

\subsubsection{Caduta}

(h > Ø) alen(us), ani, daa, paali/paale. L'unica eccezione è allaca (§4.2.1.3). Per la fricativa $[\mathrm{x}]$ cf. 4.1.

Sulla diffusione della caduta di $h$, specie nel turco balcanico, cf. Kappler (1999, 165-167). 


\subsubsection{Epentesi}

Si riscontra un caso di inserzione di $n$ in tuntun $(\rightarrow$ mazur).

\subsubsection{Metatesi}

L'unico esempio è dato da crich $(\rightarrow k a r k)$.

\subsubsection{Assimilazione}

$(\mathrm{nl}>\mathrm{n}(\mathrm{n}))$ bonar $(\rightarrow b u)$.

$(\mathrm{kç}>$ çç) aciai $(\rightarrow a c ̧ c ̧ a)$.

\subsection{Morfologia}

\subsubsection{Nome}

\subsubsection{Casi}

Troviamo corretti esempi di dativo (allaca, bona, chesmetene) e di accusativo (aciai, chesei, eisini, espapi, terezii). Un solo genitivo, pronominale: senen (=senin/senin).

\subsubsection{Suffissi possessivi}

2a pers. sing.: alen $(\rightarrow$ hal), chesmetene, ueregiein.

3a pers. sing. (accusativo): eisini.

2a pers. pl.: alenus $(\rightarrow$ hal $)$.

\subsubsection{Verbo}

\subsubsection{Tempi e modi}

Sono documentati aoristo, passato definito, imperativo, ottativo.

\section{a) Aoristo}

1a pers. sing.: al(e)run, isterũ, sairun, satáran. Per la nasale finale §4.2.1.3.

1a pers. sing., negativo: uarmezũ (=ver-), ueremẽ. È rimarchevole che accanto alla forma osmanlı classica sia attestata quella popolare, impostasi in t. moderno, in -mem (cf. anche nel Vocabulario Nuovo del 1574: istemem 'non uoglio', zechmem (= çekmem) 'non voglio menare' [Adamović 1976, 63, 64]). Il dato del 
nostro glossario sembra comunque denotare confusione con la forma di impossibilitativo veremem.

2a pers. sing.: istersĩ/isterssin/stersin.

2a pers. sing., interrogativo: aler misen, satar misin, uerir misin.

Questo tempo è usato per rendere verbi italiani al presente ('acompro', 'conto', 'uoglio/uoi/uolete'), al futuro ('contarò'), o forme desiderative ('uoglio/uoi comprare', 'uoglio/uoi uender', 'uoila dare/nõ la uoglio dare’).

\section{b) Passato definito (Belirli/görülen geçmiş zaman)}

1a pers. sing.: dedun, etun (= ettüm), saidun, satin aldun. §4.2.1.3.

2a pers. sing.: ieldin.

1a pers. pl.: etech (= ettık/ettik), smarladic.

2a pers. pl.: ieldinus.

Per gli scorretti ${ }^{\star}$ boldun, ${ }^{\star}$ boldunus $\mathrm{cf}$. il commento a $\rightarrow$ safa.

c) Imperativo

2a pers. sing.: al, atcs $(=a c ̧)$, cicar, eile, giele, gieter, sai, satin al, tart, ulg, uar, uer. 2a pers. sing., negativo: enderme, giechme (\$4.2.1.1).

3a pers. sing.: uersin.

2a pers. pl.: tuntun (§4.2.1.5).

\section{d) Ottativo (Istek kipi)}

1a pers. sing.: satin alain. La desinenza -AyIn è comune in ant. osmanlı (Deny 1921, 933; Lewis 2000, 134).

3a pers. sing.: nola. Propr. contrazione di ne ola 'quid fiat?' (Men. 5279).

1a pers. pl.: ${ }^{7}$ edelem, chidelũ (§4.2.1.1).

\subsubsection{Verbum substantivum}

1a pers. sing.: bondain, cailem.

2a pers. sing.: pecsin.

2a pers. sing., interrogativo: cail misin.

3a pers. sing.: nedr (§4.2.0.1).

7 Attribuiamo questa persona all'ottativo, come fanno la maggior parte delle grammatiche (p. es. Banguoğlu 1986, 470; Lewis 2000, 134; Ergin 2005, 311), anche se storicamente bisognerebbe considerarla piuttosto una forma d'imperativo (Deny 1921, 933). 


\subsubsection{Nome verbale in $-m A$}

Un solo esempio: uerme, che occorre certamente emendare $(\rightarrow$ gerek).

\subsection{Sintassi}

La stringatezza delle frasi permette di dire ben poco sulla sintassi, che comunque appare sostanzialmente corretta; la posizione iniziale dell'imperativo è tipica del parlato («In the colloquial, an imperative often begins a sentence», Lewis 2000, 240). L'unica curiosità da segnalare consiste nella doppia traduzione di 'misura giusto', cioè tamen ulg e ulg tamam, dove l'autore ha invertito la posizione degli elementi per motivi che ci sfuggono.

\subsection{Lessico}

Tutti gli elementi lessicali del glossario (fatte salve le modificazioni fonetiche) appartengono al vocabolario ottomano comune.

\section{Bibliografia}

\subsection{Sigle}

AngSm. = Angelico da Smirne 1955.

Men. $=$ Meninski 1680 .

Men. On.= Meninski 1687 .

\subsection{Opere}

Adamović, Milan, Ein italienisch-türkisches Sprachbuch aus den Jahren 1525-1530, Wiener Zeitschrift für die Kunde des Morgenlandes 67 (1975), 217-247.

Adamović, Milan, «Vocabulario nuovo» mit seinem türkischen Teil, Rocznik Orientalistyczny 38 (1976), 43-69.

Angelico da Smirne, Nuovo dizionario turco-italiano, Reggio Emilia, Libreria Editrice «Frate Francesco», 1955.

Banguoğlu, Tahsin, Türkçenin Grameri, Ankara, Türk Tarihi Kurumu Basım Evi, 1986.

Bombaci, Alessio, La «Regola del parlare turcho» di Filippo Argenti, Napoli, R. Istituto Superiore Orientale, 1938.

Cortelazzo, Manlio, La conoscenza della lingua turca in Italia nel '500, Il Veltro 23:2-4 (1979), 133-142.

Csató, Éva Ágnes/Johanson, Lars (edd.), The Turkic Languages, London, Routledge, 1998.

Deny, Jean, Grammaire de la langue turque (dialecte osmanli), Paris, Imprimerie Nationale, Éditions Ernest Leroux, 1921. 
Duden = Duden. Aussprachewörterbuch der deutschen Standardaussprache, bearbeitet von

Max Mangold in Zusammenarbeit mit der Dudenredaktion, Mannheim/Wien/Zürich,

Dudenverlag, 1974.

Ergenç, İclâl, Konuşma dili ve Türkçenin söyleyiş sözlüğü, İstanbul, Multilingual, 2002.

Ergin, Muharrem, Türk dil bilgisi, İstanbul, Bayrak Basım/Yayım/Tanıtım, 2005.

Hazai, György, Das Osmanisch-Türkische im XVII. Jahrhundert. Untersuchungen an den

Transkriptionstexten von Jakab Nagy de Harsány, Budapest, Akadémiai Kiadó, 1973.

Heffening, Walter, Die türkischen Transkriptionstexte des Bartholomaeus Georgievits aus den Jahren 1544-1548, Leipzig, Deutsche Morgenländische Gesellschaft, 1942.

Kappler, Matthias, Verso un nuovo Thesaurus dei turcismi balcanici: la dimensione dialettale $e$ materiale sui turcismi greco-epiroti dei secoli XVIII-XIX, in: Fusco, Fabiana/Orioles, Vincenzo (edd.), Processi di convergenza e differenziazione nelle lingue dell'Europa medievale $e$ moderna, Udine, Forum, 2000, 157-194.

Lewis, Geoffrey, Turkish Grammar, Oxford, Oxford University Press, 2000.

Lupis, Pietro, Opera nova de M. Pietro Lupis Valentiano. La qual insegna a parlare Turchesco, s.l. [Ancona], s.d. [1520 ca.] (= Adamović 1975, che ne è un riadattamento).

Meninski, Franciscus à Mesgnien, Thesaurus linguarum orientalium turcicae-arabicae-persicae. Lexicon turcico-arabico-persicum, 3 voll., Vienna, Ed. propria, 1680 (ristampa anastatica İstanbul, Simurg, 2000).

Meninski, Franciscus à Mesgnien, Complementum Thesauri linguarum orientalium, seu onomasticum latino-turcico-arabico-persicum, Vienna, Ed. propria, 1687 (ristampa anastatica İstanbul, Simurg, 2000).

Migliorini, Bruno, Storia della lingua italiana, Firenze, Sansoni, 1978.

Preindl, Joseph de, Grammaire turque d'une toute nouvelle methode d'apprendre cette langue en peu de semaines avec un vocabulaire enrichi d'anecdotes utiles et agréables, Berlin, s. ed., 1790.

Redhouse, James William, A Turkish and English Lexicon, Constantinople, A. H. Boyajian, 1890.

Rocchi, Luciano, Ricerche sulla lingua osmanlı del XVI secolo. Il corpus lessicale turco del manoscritto fiorentino di Filippo Argenti (1533), Wiesbaden, Harrassowitz, 2007.

Rocchi, Luciano, Il dizionario turco-ottomano di Arcangelo Carradori (1650), Trieste, EUT, 2011.

Rocchi, Luciano, Il «Dittionario della lingua turchesca» di Pietro Ferraguto (1611), Trieste, EUT, 2012.

Rossebastiano, Alda, La tradition des manuels polyglottes dans l'enseignement des langues, in: Auroux, Sylvain, et al. (edd.), History of the Language Sciences. An International Handbook on the Evolution of the Study of Language from the Beginnings to the Present, vol. 1, Berlin/ New York, De Gruyter Mouton, 2000, 688-698.

Stachowski, Marek, Remarks on the phonetic value of the letters $\langle y\rangle$ and $\langle\ddot{u}\rangle$ in Franciscus Meninski's Ottoman Turkish Thesaurus (1680), Studia Linguistica Universitatis lagellonicae Cracoviensis 129 (2012), 189-197.

TETTL = Tietze, Andreas, Tarihi ve etimolojik Türkiye Türkçesi lugatı / Sprachgeschichtliches und etymologisches Wörterbuch des Türkei-Türkischen, vol. 1: A-E (2002), vol. 2: F-J (2010), İstanbul et al., Simurg et al., 2002/2010.

TS = XIII. yüzyıldan beri Türkiye Türkçesiyle yazılmış kitaplardan toplanan tanıklariyle tarama sözlüğü, 8 voll., Ankara, Türk Tarihi Kurumu Basımevi, 1963-1977.

TTAS = Türkiye Türkçesi Ağızları Sözlüğü, <http://www.tdk.gov.tr/index.php?option=com_ttas\& view=ttas>.

Wendt, Heinz Friedrich, Das Fischer Lexikon. Sprachen, Frankfurt am Main, Fischer, 1987. 